\title{
Improvements on the fundamental parameters of the open cluster Tombaugh 1 through Washington system photometry
}

\author{
A. E. Piatti ${ }^{1}$, J. J. Clariá ${ }^{2}$, and A. V. Ahumada ${ }^{2}$ \\ 1 Instituto de Astronomía y Física del Espacio, CC 67, Suc. 28, 1428, Buenos Aires, Argentina \\ 2 Observatorio Astronómico, Universidad Nacional de Córdoba, Laprida 854, 5000 Córdoba, Argentina \\ e-mail: [claria; andrea]@mail.oac.uncor.edu
}

Received 8 January 2004 / Accepted 31 March 2004

\begin{abstract}
We present CCD photometry in the Washington system $C$ and $T_{1}$ passbands down to $T_{1} \sim 18.5$ mag in the field of Tombaugh 1, a little studied open cluster located in the third Galactic quadrant. We measured $T_{1}$ magnitudes and $C-T_{1}$ colours for a total of 1351 stars distributed throughout an area of $13.6 \times 13$ '. $^{\prime}$. A cluster radius of 4.3 0 $^{\prime} .3$ was estimated from star counts in 100-pixel a side boxes distributed throughout the entire observed field. Based on the best fits of isochrones computed by the Geneva group for $Z=0.008$ to the $T_{1}$ vs. $C-T_{1}$ colour-magnitude diagram, we derive a colour excess $E\left(C-T_{1}\right)=$ $0.55 \pm 0.10$, equivalent to $E(B-V)=0.30 \pm 0.05$, a distance of $(2.2 \pm 0.5) \mathrm{kpc}$ from the Sun and an age of $1.3_{-0.2}^{+0.1} \mathrm{Gyr}$. The latter value is in good agreement with that derived from the independent metallicity $\delta T_{1}$ index defined in Geisler et al. (1997, AJ, 114, 1920). An independent metallicity estimation using the $\left[M_{T_{1}},\left(C-T_{1}\right)_{0}\right]$ plane with the standard giant branches of Geisler \& Sarajedini $(1999, \mathrm{AJ}, 117,308)$ yields $[\mathrm{Fe} / \mathrm{H}]=-0.30 \pm 0.25 \mathrm{dex}$, a value which lends support to the one obtained from the isochrone fit. Tombaugh 1 is then found to be a relatively metal-poor intermediate-age open cluster.
\end{abstract}

Key words. Galaxy: open clusters and associations: individual: Tombaugh 1 - Galaxy: general - techniques: photometric

\section{Introduction}

As is well known, intermediate-age and old Galactic open clusters range widely in terms of distance, metallicities and ages (Friel 1995; Friel et al. 2002). They are located in the disc of the Galaxy and are extremely useful as probes of both age and metallicity in the dynamical and chemical evolution of the Galactic disc. Research dealing with possible abundance gradients in the disc and the age-metallicity relationship for the Galaxy (e.g., Strobel 1991; Chen et al. 2003) necessitates highquality data on the greatest possible number of clusters ranging vastly in age.

The present work is part of an ongoing project of observation of some unstudied or poorly studied open clusters, located in different regions of the Galaxy. Our purpose is to obtain good-quality CCD photometric data not only to produce a larger sample of studied open clusters, but also to derive their basic properties accurately. We have already reported results on the relatively young and metal-poor open clusters NGC 2194 and NGC 2324 (Piatti et al. 2003a, 2004a), on the intermediateage cluster NGC 2627 (Piatti et al. 2003b) and on the old-metalpoor anticentre cluster Trumpler 5 (Piatti et al. 2004b).

The faint open cluster Tombaugh 1, IAU designation C0658-204, is located in a moderately rich star field in

Send offprint requests to: A. E. Piatti, e-mail: andres@iafe.uba.ar
Canis Major at $\alpha=7^{\mathrm{h}} 00^{\mathrm{m}} 29^{\mathrm{s}}, \delta=-20^{\circ} 34^{\prime}(2000.0)$ and Galactic coordinates $l=232^{\circ} .22, b=-7.32$. This cluster, together with Tombaugh 2 (C0701-207), was discovered by Tombaugh (1938), during his search for trans-Neptunian planets. Tombaugh 1 was additionally rediscovered about 20 years later both by Haffner (1957) and by Tifft (1959), in the latter case as a product of a search for open clusters near classical Cepheid variables. Tombaugh (1941) presented for the first time photographic plates of both clusters, along with three other objects discovered in the meantime. He observes that Tombaugh 1 has an angular radius of 5.0, and that it is probably obscured by the presence of numerous field stars.

Tombaugh 1 has been the subject of very few previous studies. Ruprecht (1966) considered it to belong to Trumpler (1930) class III $2 \mathrm{~m}$, i.e. a moderately populated open cluster with no noticeable concentration and a medium range in the brightness of the stars. Turner (1982) obtained photoelectric photometry for 26 stars in the cluster field in order to analyse the membership of the Cepheid star XZ CMa. He derived a colour excess $E(B-V)=0.27 \pm 0.01 \mathrm{mag}$, a distance of $1.26 \mathrm{kpc}$ from the Sun, and an age of $800 \mathrm{Myr}$. He also underlined the presence of several subgiant and giant stars, characteristics of a moderately old cluster, while Ahumada \& Lapasset (1995) identified one star (Turner's No. 2) as a good candidate for blue straggler. More recently, Dutra \& Bica (2000) derived a far-infrared 


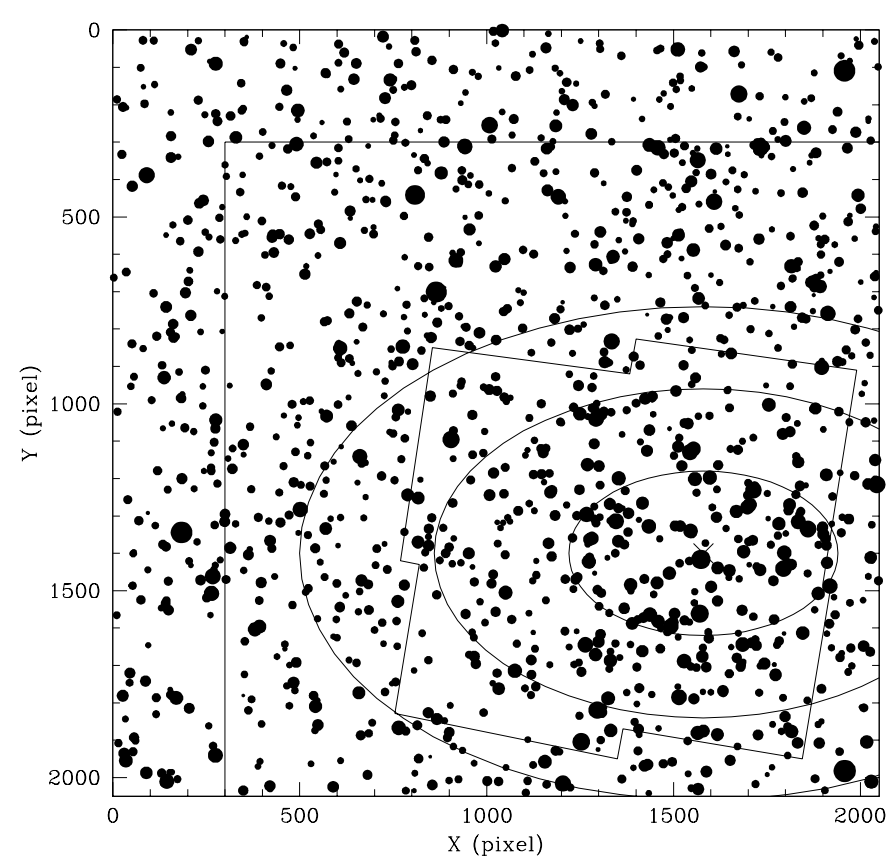

Fig. 1. Schematic finding chart of the stars observed in the field of Tombaugh 1. North is up and East is to the left. The sizes of the plotting symbols are proportional to the $T_{1}$ brightness of the stars. Three concentric ellipses around the cluster centre, the adopted star field and the field observed by Carraro \& Patat (1995) are also drawn. Details are described in Sect. 3.

reddening $E(B-V)_{\mathrm{FIR}}=0.40$ for Tombaugh 1 from DIRBE/IRAS $100 \mu \mathrm{m}$ dust emission in the cluster field. Even when the angular radius of Tombaugh 1 renders it appropriate for CCD camera analysis, the only CCD photometric study up to date was performed by Carraro \& Patat (1995, hereafter CP95), who obtained VI photometry within a field of about $8^{\prime} \times 8^{\prime}$. They estimated as $0.40 \mathrm{mag}, 3.0 \mathrm{kpc}$ and $\sim 1 \mathrm{Gyr}$ the reddening, the distance and the age of Tombaugh 1 , values which show overt disagreement with the ones obtained by Turner (1982). The CP95 colour-magnitude diagram (CMD) reveals a clear main sequence down to $V \approx 20.0 \mathrm{mag}$, with the turn-off located at $V \approx 14.5 \mathrm{mag},(V-I) \approx 0.6 \mathrm{mag}$. Neither the morphology of the turn-off nor the evolved region of the CMD is clearly defined, thus making it impossible to ascertain whether or not a cluster giant branch really exists. Incidentally, one of the factors motivating the present study is that CP95 do make reference to a series of problems in their analysis and their results which they have been unable to explain in a satisfactory manner. For instance, they have not managed to produce an adequate fitting of theoretical isochrones, nor do they succeed in quantitatively determining the influence of possible sources of broadening of the main sequence, even when they do mention them in general terms. As they admit it, the lack of any knowledge about the cluster metal content is the main obstacle hindering a suitable fit of the cluster sequence with the theoretical isochrones.

A further factor warranting the current investigation is that the $C$ and $T_{1}$ filters of the Washington system (Canterna 1976) are far more effective than the $V$ and $I$ filters in determining age of a star cluster by means of theoretical isochrones. In fact, as shown by Geisler \& Sarajedini (1999), the metallicity sensitivity of their standard giant branches (SGBs, each giant branch corresponds to an isoabundance curve) in the $M_{T_{1}}$ vs. $\left(C-T_{1}\right)_{0}$ plane is three times higher than that of the VI technique (Da Costa \& Armandroff 1990) and, consequently, it is possible to determine metallicities three times more precisely for a given photometric error. Even though the SGBs were defined for $[\mathrm{Fe} / \mathrm{H}]<-0.5$ using globular clusters older than 10 Gyr, Geisler et al. (2003) have recently derived the correction to be applied to the SGB metallicities as a function of age.

In the present study, we report the results obtained from CCD photometry in the $C$ and $T_{1}$ passbands of the Washington system up to $T_{1} \sim 18.5 \mathrm{mag}$ in the field of Tombaugh 1 . These data are employed in order to make a new and independent determination of reddening, distance, age and metallicity. In Sect. 2 we present the observational material, while in Sect. 3 we describe the features of the observed field and determine the cluster centre and its angular extension. In Sect. 4 the main features of the observed CMD are described and the influence of the different sources of dispersion in the cluster MS is discussed. In Sect. 5 and through the fitting of theoretical isochrones computed for the Washington system, we determine the fundamental cluster parameters and compare them with previous estimates. A short summary of our main conclusions is given in Sect. 6.

\section{The data: Washington photometry}

Images of the cluster field were obtained using the $2048 \times$ 2048 pixel Tektronix $2 \mathrm{~K} \mathrm{\# 3} \mathrm{CCD} \mathrm{at} \mathrm{the} \mathrm{prime} \mathrm{focus} \mathrm{of} \mathrm{the}$ Cerro Tololo Inter-American Observatory (CTIO) 0.9-m telescope on the night of 1997 December 23. The detector used has a pixel size of $24 \mu \mathrm{m}$, producing a scale on the chip of 0.40 pixel $^{-1}$ (focal ratio f/13.5) and a total field size of $13.6 \times 13.6$. Note that the CCD field is nearly 4 times the size of the field covered by $\mathrm{CP} 95$, and should thus provide a much larger star sample. The only 26 stars observed by Turner (1982) are distributed within a circle of $10^{\prime}$. We employed a gain of $5 \mathrm{e}^{-} /$ADU which yielded a readout noise of about $4 \mathrm{e}^{-}$. The CCD was controlled by the CTIO Arcon 3.3 data acquisition system in the QUAD amplifier mode.

The images were taken with the Washington system $C$ and $T_{1}$ filters. The recommended prescription for the $C$ filter that we utilized is the one proposed in Geisler (1996). Photometric conditions prevailed during the observing night and images of Tombaugh 1 and of numerous standard stars from the lists of Landolt (1992) and Geisler (1996), covering a wide range in colour, were obtained. We took a series of $2 \times 10$-s $T_{1}$ exposures and $2 \times 45$-s $C$ exposures for Tombaugh 1 . Figure 1 shows a schematic finding chart of the observed cluster field, in which the sizes of the plotting symbols are proportional to the $T_{1}$ brightness of the stars. Some bright stars within the observed area were not drawn because they saturated the chip. According to the CMDs of Turner (1982) and Carraro \& Patat (1995), they should be field stars. Some few relatively fainter stars placed in bad pixels or below the wing of bright stars were not measured either. 
Table 1. CCD $C T_{1}$ data of stars in the field of Tombaugh 1.

\begin{tabular}{cccccccc}
\hline \hline Star & $\begin{array}{c}X \\
\text { (pixel) }\end{array}$ & $\begin{array}{c}Y \\
\text { (pixel) }\end{array}$ & $\begin{array}{c}T_{1} \\
(\mathrm{mag})\end{array}$ & $\begin{array}{c}\sigma\left(T_{1}\right) \\
(\mathrm{mag})\end{array}$ & $\begin{array}{c}C-T_{1} \\
(\mathrm{mag})\end{array}$ & $\begin{array}{c}\sigma\left(C-T_{1}\right) \\
(\mathrm{mag})\end{array}$ & $n$ \\
\hline. &. &. &. &. &. &. &. \\
326 & 1391.877 & 510.788 & 17.618 & 0.069 & 1.608 & 0.074 & 2 \\
327 & 398.651 & 510.892 & 17.008 & 0.005 & 1.364 & 0.011 & 2 \\
328 & 1967.316 & 512.614 & 16.483 & 0.009 & 1.475 & 0.003 & 2 \\
329 & 549.964 & 518.922 & 16.772 & 0.029 & 1.277 & 0.083 & 2 \\
330 & 1390.155 & 519.028 & 18.222 & 0.101 & 1.578 & 0.127 & 1 \\
. &. &. &. &. &. &. &. \\
. &. &. &. &. &. &. &. \\
. &. &. &. &. &. &. &. \\
\hline
\end{tabular}

NOTE: $(X, Y)$ coordinates correspond to the reference system of Fig. 1. Magnitude and colour errors are the standard deviations of the mean, or else the observed photometric errors for stars with one measurement.

Each frame was trimmed, bias-subtracted and flat-fielded using domeflat exposures per filter to calibrate the CCD instrumental signature. The $C T_{1}$ images were reduced at CTIO, using QUADPROC tasks of the IRAF ${ }^{1}$ software package. Instrumental magnitudes of the standard and Tombaugh 1 fields were obtained at the Instituto de Astronomía y Física del Espacio (IAFE, Argentina) with stand-alone versions of DAOPHOT and ALLSTAR kindly provided by Peter B. Stetson. Aperture corrections were determined from PSF stars distributed throughout the chip, the typical value being -0.01 . The programme star instrumental magnitudes were then transformed onto the standard Washington system via the aperture photometry of numerous standard stars. The transformation equations we obtained are:

$$
\begin{aligned}
c_{i}= & (2.068 \pm 0.030)+T_{1}+\left(C-T_{1}\right)-(0.060 \pm 0.010) \\
& \times\left(C-T_{1}\right)+(0.308 \pm 0.015) \times X_{i}, \\
t_{1_{j}}= & (2.930 \pm 0.022)+T_{1}+(0.066 \pm 0.008) \times\left(C-T_{1}\right) \\
& +(0.104 \pm 0.011) \times X_{j},
\end{aligned}
$$

where capital and lower-case letters represent standard and instrumental magnitudes, respectively, and $X$ is the effective airmass at the midpoint of the observation of the $i$, jth standard star. The rms scatter of the standard stars was 0.017 and 0.013 for $C$ and $T_{1}$, respectively, indicating the night was of good photometric quality.

We generated a master table containing a running number, the $X$ and $Y$ coordinates, the $T_{1}$ magnitudes and $C-T_{1}$ colours, the observational errors $\sigma\left(T_{1}\right)$ and $\sigma\left(C-T_{1}\right)$ provided by DAOPHOT and the number of observations. This table was built by combining all the independent measurements using the stand-alone DAOMATCH and DAOMASTER programmes kindly provided by Peter B. Stetson. Table 1 gives this information. Only a portion of this table is shown here for guidance regarding its form and content, its whole content being available upon request to the first author of this paper. The photometric errors

${ }^{1}$ IRAF is distributed by the National Optical Astronomy Observatories, which is operated by the Association of Universities for Research in Astronomy, Inc., under contract with the National Science Foundation.

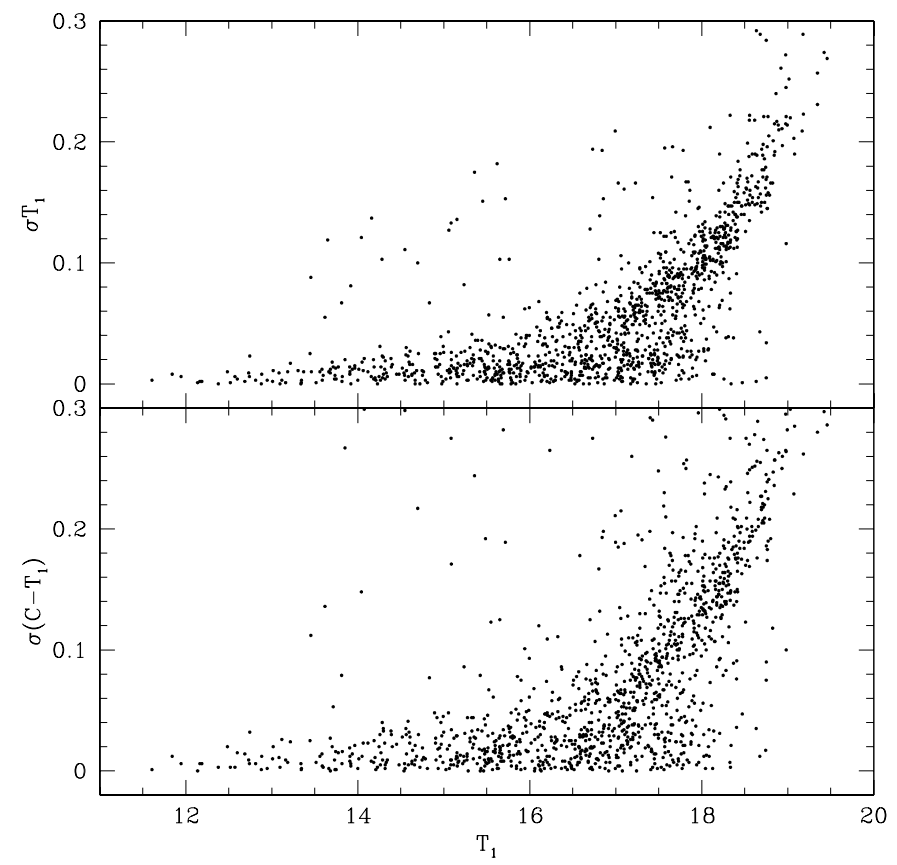

Fig. 2. Magnitude and colour photometric errors as a function of $T_{1}$.

provided by DAOPHOT for the $T_{1}$ magnitude and $C-T_{1}$ colours have been plotted against their corresponding $T_{1}$ magnitudes in Fig. 2.

\section{Features of the observed field}

We included in Fig. 1 all stars measured, at least once, with the $C$ and $T_{1}$ filters. Each star was plotted using a filled circle of a size proportional to its brightness in the $T_{1}$ passband. As can be inferred from the higher concentration of stars at the lower right quarter of the figure, the telescope was not centred on the cluster itself but slightly northeast in order to avoid some of the bright stars to the south and west of the cluster. The position of the cluster on the chip allowed us to devote an important fraction of the observed area to sample its surrounding field. Note also that our photometry covers an area around the cluster approximately four times that covered by the observations of CP95, as can be seen from the parallelepiped drawn 
in Fig. 1, which frames the field they focused on. They also observed a field located about $15^{\prime}$ northwards from the cluster for comparison purposes. As a result of the larger cluster field coverage in combination with the wider wavelength baseline of the $C T_{1}$ photometry, we observed around 40 per cent more stars than CP95.

Tombaugh 1 is easily identifiable within a little crowded star field. However, since one can mistake distinct small groups of stars for the cluster centre, we decided to determine the cluster central position statistically. The strategy to carry out that task consists in tracing the stellar density profiles projected onto the directions of the $X$ and $Y$ axes, and in obtaining the coordinates associated to the peak of the stellar density distribution by fitting those profiles. In order to build the $X$ projected density profile, we counted the number of stars distributed along fringes of a fixed width and oriented along the $Y$ axis. Similarly, the $Y$ projected density profile was constructed using fringes placed along the $X$ axis. The width of the fringes was fixed in 100 pixels for both axes. This value results in a compromise between minimizing spurious effects mainly caused by the presence of localized groups, rows or columns of stars, and maximizing the spatial resolution. The range of useful bin sizes is also constrained by the mean field stellar density, which translates into a lower limit for the mean free path between two stars. In our case, bin sizes smaller than 60 pixels do not provide any additional information but only statistical noise.

The projected stellar density profiles were fitted using the NGAUSSFIT routine of the STSDAS IRAF package. We chose the multiple gaussian fitting option and fixed the constant and linear terms to the corresponding background level and to zero, respectively, and set the number of matching gaussians to one. The amplitude, the full width at half maximum (FWHM) and the centre of the Gaussian acted as variables. We iterated the fitting procedure once in average, after eliminating a couple of dispersed points. The final coordinates for the cluster centre turned out to be $\left(X_{\mathrm{C}}, Y_{\mathrm{C}}\right)=(1580 \pm 30,1400 \pm 30)$ pixels, which were adopted in the following analysis. The cluster centre is marked by a cross in Fig. 1. We also derived the FWHMs of the projected stellar density profiles. We obtained $\sigma(X)=360 \pm$ 45 pixels and $\sigma(Y)=220 \pm 40$ pixels for the half widths at half maximum of the projected gaussians in the $X$ and $Y$ directions, respectively. We drew three ellipses in Fig. 1 centred on the cluster with semi-major and semi-minor axes parallel to the $X$ and $Y$ axes, respectively, and with sizes once, twice and three times $\sigma(X)$ and $\sigma(Y)$. As can be seen, the cluster appears to be elongated in the east-west direction.

We then built the cluster radial profile with the aim of: (i) estimating the cluster radius generally used as an indicator of the cluster dimension; (ii) evaluating the difference in determining the cluster size from radial star counts from its centre instead from star counts of the whole field projected onto perpendicular axes; and (iii) establishing the area out of which field stars practically prevail. The availability of a field area is highly valued, mainly because of the advantages in disentagling fiducial cluster and field features in the observed colour-magnitude diagram (CMD). The most common way of building the cluster stellar density radial profile consists in counting the number of stars distributed in concentric rings

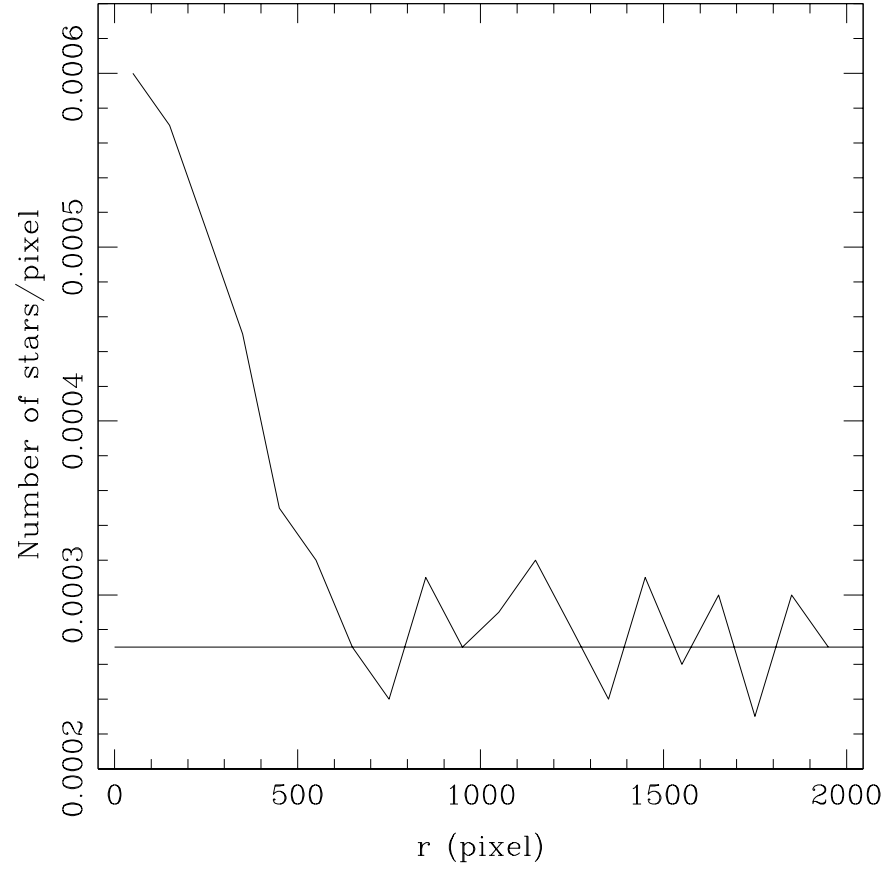

Fig. 3. Stellar density profile centred at $\left(X_{\mathrm{C}}, Y_{\mathrm{C}}\right)=(1580,1400)$ pixels for stars observed in the field of Tombaugh 1 . The horizontal line represents the background level measured in the field area.

around the cluster centre and normalizing the sum of stars in each ring to the unit area. This procedure allows the observer to easily stretch the radial profile as much as he can until complete circles can be traced in the observed field. However, so as to move even further away from the cluster centre, we decided to follow another method based on counts of stars located in boxes of 100 pixels a side distributed throughout the whole field. Thus, the number of stars per unit area at a given radius $r$ can be directly calculated through the expression:

$\left(n_{r+50}-n_{r-50}\right) /\left(\left(m_{r+50}-m_{r-50}\right) \times 100^{2}\right)$,

where $n_{j}$ and $m_{j}$ represent the number of counted stars and boxes of 100 pixels a side included in a circle of radius $j$. The resulting stellar density profile is shown in Fig. 3. According to the figure, the fringes $0<X<300$ pixels and $0<Y<300$ pixels are placed far enough from the cluster region to allow us to consider them sky areas whose stars model the characteristics of the surrounding cluster field in the CMD. We adopted this region in the subsequent analysis as the "star field area", as is also indicated in Fig. 1. By taking advantages of the relative extension of the field area, we estimated a mean field stellar density of 0.00027 pixels $^{-1}$. This density results slightly lower than half of the central cluster density. The horizontal line in Fig. 3 represents the derived background level, which is in very good agreement with the relatively extended stretch of the field stellar density distribution computed radially from the cluster centre. From this figure, we also estimated a cluster radius of $(650 \pm 50)$ pixels, equivalent to $4.3 \pm 0 ! 3$. This value is close to the size of the semi-major axis of the central ellipse of Fig. 1, as well as to the boundary of the field observed by CP95. Turner (1982) performed star counts in concentric rings and found nuclear and coronal radii of $5^{\prime}$ and $10^{\prime}$, respectively. We note, 


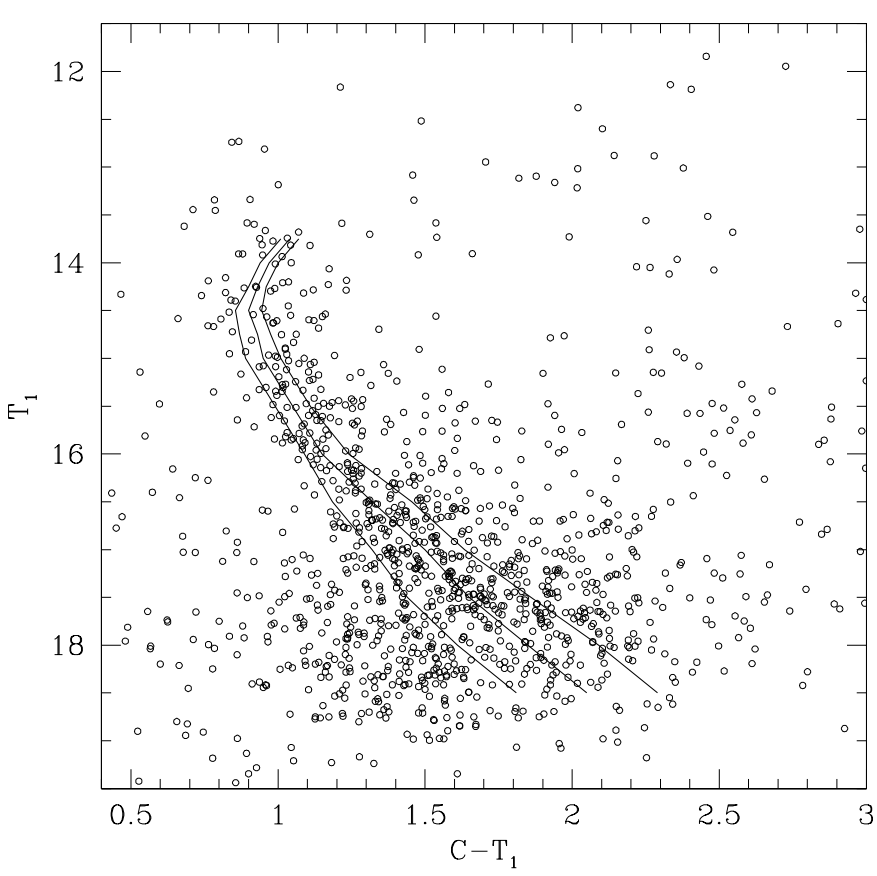

Fig. 4. $\left(T_{1}, C-T_{1}\right)$ colour-magnitude diagram for stars observed in the field of Tombaugh 1. The fiducial cluster MS and the $\pm 3 \times \sigma\left(C-T_{1}\right)$ shifted fiducial MSs are overplotted.

however, that even using stars down to four magnitudes fainter than those used by Turner, we did not find any evidence for a poorly-populated and extended corona.

\section{The $T_{1}$ vs. $C-T_{1}$ colour-magnitude diagram of Tombaugh 1}

The CMD for the stars plotted in Fig. 1 is depicted in Fig. 4. The figure shows that the cluster main sequence (MS) is the most revelant feature. At its brightest part it seems somewhat broadened not only by the presence of possible field stars and/or cluster binaries but also by intrinsic scattering. At intermediate magnitudes, the cluster MS presents a couple of gaps of stars that resemble those caused by evolutionary effects, although dynamical reasons cannot be ruled out (Turner 1996). Particularly, one of the clearest gaps we roughly identified is at $T_{1} \approx 15.8$ and $C-T_{1} \approx 1.2$. From $T_{1}=17.0$ $(\simeq V=18.0)$ down to the faintest reached magnitude, the field contamination remarkably blurs the cluster MS. The fact that most of the field stars are predominantly distributed in a colour range similar to that of the cluster MS and share the fainter magnitudes of the CMD with the cluster stars, suggests that the field contamination mainly comes from foreground stars (their reddenings are of the order of the cluster reddening). They belong to a disk component older than the cluster (see, e.g., $\mathrm{Ng}$ et al. 1996). Finally, the CMD also shows a handful of stars distributed along what could be associated to the cluster red giant clump (RGC) and its giant branch (GB), and even some probable sub-giant and blue straggler stars. These characteristics suggest that the age of Tombaugh 1 could be around that of an intermediate-age open cluster.
With the aim of distinguishing between the different sources of dispersion in the cluster MS and determining their degrees of influence, we started taking into account the photometric errors and the field star contamination. An inspection of Table 1 shows that 64 per cent of the total number of measured stars have two measures of their $T_{1}$ magnitudes and $C-T_{1}$ colours, while the remaining 36 per cent have only one measure in both $C$ and $T_{1}$ filters. For those stars with two measures, we averaged the individual magnitudes and colours and adopted the rms errors as photometric errors. These stars mainly expand from the top of the CMD down to $T_{1}=18.5$, and cover the whole extension of the cluster MS. For those stars with only one measure, we directly adopted the errors provided by ALLSTARS. These stars mainly define the lower part of the CMD and are found from $T_{1}=17$ down to the limiting magnitude. Typically, stars with two measures have in average $\sigma\left(T_{1}\right)<0.015$ and $\sigma\left(C-T_{1}\right)<0.02$ for $T_{1}<16$. Half of those stars lying between $T_{1}=16$ and 18.5 present the same uncertainties as the brighter stars, while the other half has $\left\langle\sigma\left(T_{1}\right)\right\rangle \approx\left\langle\sigma\left(C-T_{1}\right)\right\rangle \approx 0.06$. For stars with one measure, $\sigma\left(T_{1}\right)$ and $\sigma\left(C-T_{1}\right)$ increase exponentially from 0.04 and 0.06 up to 0.2 and 0.26 , respectively, for $T_{1}$ between 17 and 19. In order to illustrate the effect of the photometric errors in the cluster MS, we included in Fig. 4 the fiducial cluster $\mathrm{MS}$ and two additional fiducial MSs shifted by $\pm 3 \times \sigma\left(C-T_{1}\right)$. Note that both curves fall nearly within the overall dispersion of the cluster MS. The fiducial cluster MS was built by joining the points on the cluster MS with the highest star densities.

To remove the field stars from the cluster CMD, we tried to statistically reproduce the field CMD with stars distributed within the cluster area. Figures $5 \mathrm{a}$ and $5 \mathrm{~b}$ show the cluster and field CMDs, respectively. These CMDs were built using all the stars distributed in the largest ellipse of Fig. 1 and in the field area defined in Sect. 3. We would like to remark that we could have also used circular extractions in Fig. 1, but preferred the elliptical ones since they trace the contour of different isodensity levels more accurately. The difference between the lower envelopes of cluster and field MSs become thus evident, being the field MS a tilted straight star sequence. To obtain a CMD analogous to that of the field, we first counted the number of stars in bins of $\left(\Delta\left(T_{1}\right), \Delta\left(C-T_{1}\right)\right)=(0.5,0.1)$ mag in the field CMD and normalized them to the unit area. The chosen sizes for $\Delta\left(T_{1}\right)$ and $\Delta\left(C-T_{1}\right)$ come from previous iterations of the cleaning process until reaching the best values which produced the highest number of details in the cluster CMD and retained the lowest number of field stars. Then, we calculated the number of stars expected to be found in each $\left(\Delta\left(T_{1}\right)\right.$, $\Delta\left(C-T_{1}\right)$ ) box in the cluster area (we used as cluster area the observed field without the field area), and selected from it the computed amount of stars for the respective $\left(\Delta\left(T_{1}\right), \Delta\left(C-T_{1}\right)\right)$ intervals. By subtracting the selected stars from Fig. 5a, we obtained the CMD shown in Fig. 5c. Although some interlopers still remain, the observed cluster CMD was satisfactorily cleaned and the cluster MS is now clearly shown. Its broadness may be due mainly to evolutionary effects, as well as to the presence of binaries, since the distribution of stars around the MS is preferentially towards higher luminosities at a fixed colour. These are just the effects that binaries of various mass 


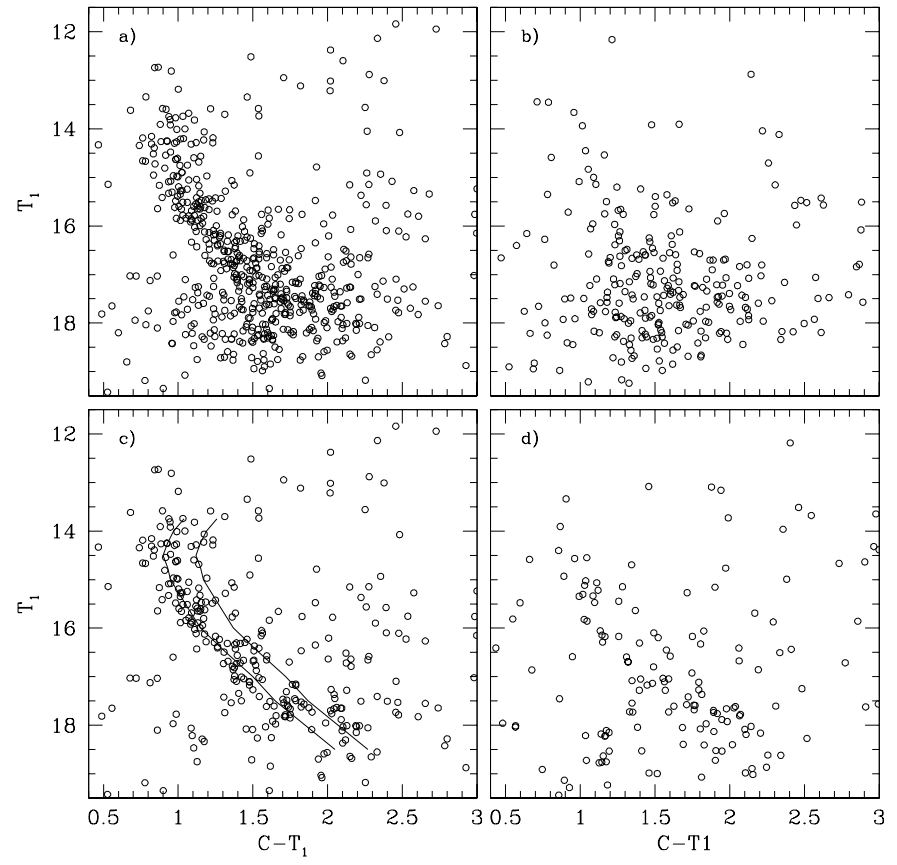

Fig. 5. $\left(T_{1}, C-T_{1}\right)$ colour-magnitude diagram for stars observed in different extracted regions: a) the largest ellipse of Fig. 1; b) field area; c) same as in a) with field stars subtracted. The fiducial cluster MS and the Burki's limit shifted fiducial MS are overplotted ; d) the region delimited by contours of a) and b). See Sect. 4 for details.

ratios would have on the CMD. As it is well known, the occurrence of binary stars in open clusters is not a rare phenomenon (see, e.g., Mermilliod \& Mayor 1989, 1990). In Fig. 5d we plotted the field subtracted CMD for stars placed outside of both the largest ellipse and of the field area. The figure shows that beyond the cluster radius, in the coronal cluster region, the cluster features appear to be quite tenuous. For this reason and in order to derive the cluster fundamental parameters, we used the CMD of Fig. 5c combined with 15 red giants observed in the range $C-T_{1}>1.5$ and $T_{1}<13.5$.

\section{Cluster fundamental parameters}

It is well known that the cluster metallicity plays an important role when estimating its age from the fit of theoretical isochrones. Indeed, theoretical isochrones with the same age but with different metallicities can range from slightly to remarkably distinguishable depending on their sensitivities to metallicity. For example, the $T_{1}$ vs. $C-T_{1} \mathrm{CMD}$ have nearly three times the metallicity sensitivity of the $V$ vs. $V-I$ CMD (Geisler \& Sarajedini 1999). The distinction is particularly evident for the evolved phases of the RGC and GB. As far as zero age main sequences (ZAMSs) are concerned, they are often less affected by metallicity effects, and can even exhibit imperceptible variations for a specific metallicity range within the photometric errors. This is the case of ZAMSs of Galactic open clusters, among them Tombaugh 1 . Since there is no previous estimate of the cluster metal content available, we followed the general rule of starting by adopting both solar and sub-solar values for the cluster metal content.
As for the isochrone set, we used those computed by the Geneve group (Lejeune \& Schaerer 2001) which are available with overshooting effect in steps of $\Delta \log t=0.05$ dex. According to previous studies (see, e.g., Piatti et al. 2003c), these isochrones lead to similar results to those derived from the Padova group's isochrones (Girardi et al. 2000). Then, we first fitted the ZAMSs for $Z=0.02$ and 0.008 to the cluster CMD and derived colour excesses and apparent distance moduli differing in $\Delta\left(E\left(C-T_{1}\right)\right)=0.10$ and $\Delta\left(T_{1}-M_{T_{1}}\right)=0.25$. Since the fits are performed through the lower envelope of the cluster MS, the presence of binaries practically does not affect the choice of the best fit isochrones. The resulting values of $E\left(C-T_{1}\right)$ and $T_{1}-M_{T_{1}}$ for the solar metallicity make the cluster less reddened and more distant from the Sun. Notice that had we adopted the above mentioned differences as uncertainties in the determination of the reddening and the distance modulus of the cluster, any ZAMS would be shifted within the thickness of the cluster MS. The colour excess and apparent distance modulus obtained for $Z=0.008$ are $E\left(C-T_{1}\right)=0.55 \pm 0.10$ and $T_{1}-M_{T_{1}}=12.50 \pm 0.25$. By using $E\left(C-T_{1}\right) / E(B-V)=1.97$ and $T_{1}-M_{T_{1}}=$ $V-M_{V}-0.58 \times E(B-V)$ (Geisler et al. 1996), we derived $E(B-V)=0.30 \pm 0.05$ and $V-M_{V}=12.70 \pm 0.30$, which places the cluster at a distance $d$ of $(2.2 \pm 0.5) \mathrm{kpc}$ from the Sun. The distance error was calculated through the expression $\sigma(d)=0.46 \times\left[\sigma\left(V-M_{V}\right)+3.2 \times \sigma(E(B-V))\right] \times d$, where $\sigma\left(V-M_{V}\right)$ and $\sigma(E(B-V))$ represent the estimated errors in $V-M_{V}$ and $E(B-V)$, respectively.

Burki (1975) has studied CMDs of cluster fields affected by differential reddening and estimated that the lower limit for the MS width in such cases is given by $\Delta(B-V)=0.11 \mathrm{mag}$. This means that cluster MS stars located beyond 0.11 mag in $B-V$ from the ZAMS are statistically affected by a larger amount of interstellar extinction than the mean cluster reddening. The Burki's limit is equivalent to $\Delta\left(C-T_{1}\right)=0.22 \mathrm{mag}$ for the $C-T_{1}$ colour, this value being nearly twice as big as the error of our $E\left(C-T_{1}\right)$ estimate. By using this value, we shifted the fiducial cluster MS redwards and depicted the result in Fig. 5c. As can be seen, far from any evidence of differential reddening across the cluster field, Tombaugh 1 appears to be affected by a uniform interstellar reddening. The noteworthy variations of the width of the cluster MS along the $T_{1}$ magnitude are mainly due both to evolutionary effects and to the presence of binaries. We identified gaps of stars centred at $\left(T_{1}, C-T_{1}\right) \approx(15.8$, $0.85),(16,1.25)$ and $(17.2,1.65)$, some of which have also been detected in other intermediate-age and old open clusters (see, e.g., Bonifazi et al. 1990; Bergbusch et al. 1991; Park \& Lee 1999). Although there is not yet any satisfactory explanation for these gaps, they seem to be related to the existence of convective overshooting in the core of moderate mass stars before and during the hydrogen exhaustion phase (Crowe \& Mitalas 1982; Lee 1997).

We then estimated the age of the cluster from the metallicity independent $\delta T_{1}$ index, which measures the difference in $T_{1}$ magnitude between the RGC and the MS turn-off (TO). We measured $T_{1}=13.10 \pm 0.10$ and $T_{1}=13.60 \pm 0.15$ for the RGC and TO, respectively, hence $\delta T_{1}=0.50 \pm 0.25$, which implies a cluster age of $(1.0 \pm 0.3)$ Gyr (Geisler et al. 1997). 


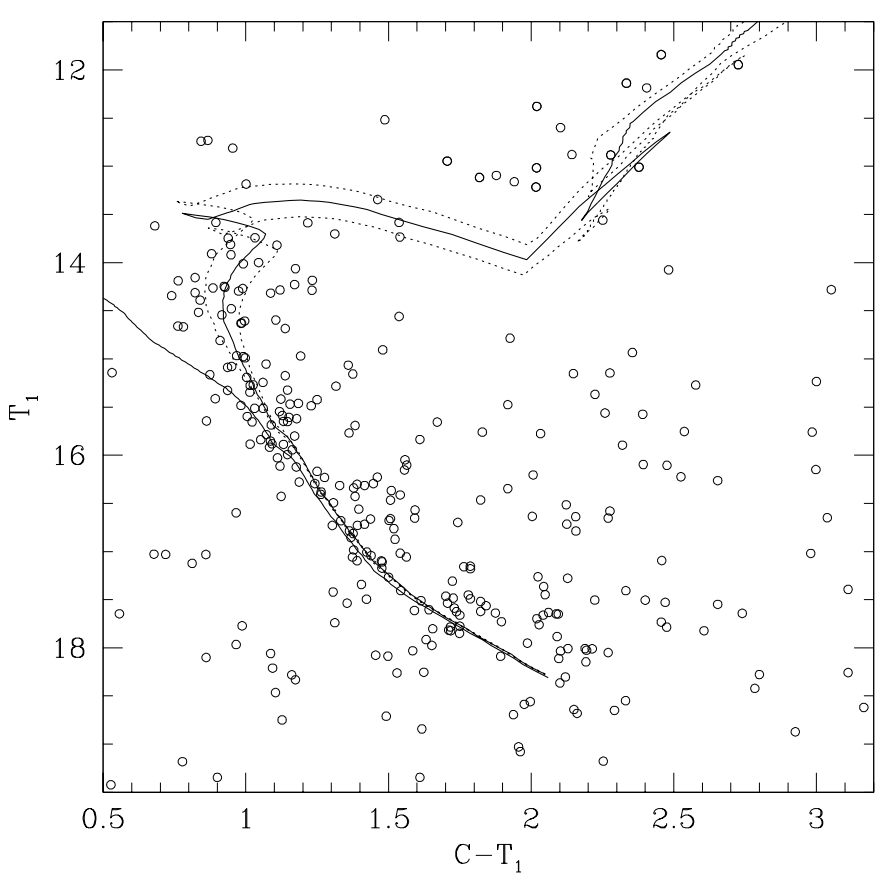

Fig. 6. Field stars cleaned $\left(T_{1}, C-T_{1}\right)$ CMD of Tombaugh 1 . The zero age main sequence and the isochrones for $\log t=9.05,9.10$ and 9.15 $(Z=0.008)$ from Lejeune \& Schaerer (2001), computed taking into account overshooting, are overplotted.

Note that we assigned to the TO $T_{1}$ magnitude an uncertainty fifteen times as much larger than that typical of the photometry at the TO level. Despite the somewhat disperse nature of the TO (see Fig. 1 of Phelps et al. 1994, for a better visualization of the position of the defined TO), the rather sparse RGC and especially some quota of subjectivity in this procedure, we used the resulting age only as reference for focusing on the selection of theoretical isochrones. We performed the fit of the cluster CMD using isochrones with $Z=0.02$ and $Z=0.008$, shifted them by the previously determined $E\left(C-T_{1}\right)$ colour excess and $T_{1}-M_{T_{1}}$ apparent distance modulus. The difference between the isochrones of both metallicities which best resemble the cluster features turns out to be $\Delta t=0.3 \mathrm{Gyr}$, the isochrone with solar metal content being younger. Both isochrones reproduce in the same way the cluster MS, from the faintest magnitudes until the TO, but they differentiate each other in the locus of the RGC and GB. For the isochrone of $Z=0.008$, these features are roughly located 0.20 mag closer to the observed ones. The cluster age derived for $Z=0.008$ is $t=1.3_{-0.2}^{+0.1}$ Gyr. In Fig. 6 we show the $T_{1}$ vs. $C-T_{1}$ cluster CMD with the ZAMS and the isochrone of $\log t=9.10$ for $Z=0.008$ superimposed (solid lines). For comparison purposes we represented with dashed lines two isochrones of log $t=9.05$ and $9.15(Z=0.008)$, respectively. Note that the largest discrepancies betweem the observed CMD and the theoretical isochrones occur at the red giant phase. The theoretical RGC has an offset of $\Delta\left(C-T_{1}\right) \sim 0.40$ mag with respect to the position of the observed RGC. This offset is of the same order of those noticed in different studies of open clusters using the BVI Johnson-Counsins photometric system, and some of them are quoted by Piatti et al. (2004b).

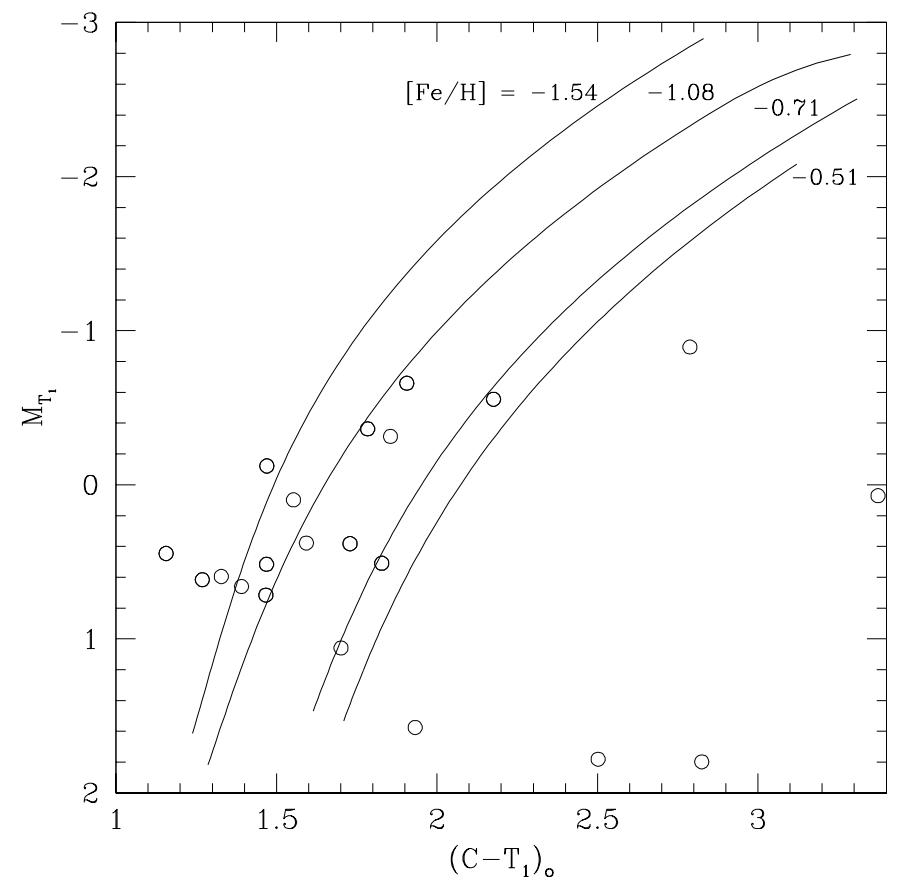

Fig. 7. Washington $M_{T_{1}}$ vs. $\left(C-T_{1}\right)_{0}$ diagram for stars in the cluster giant region, with standard giant branches from Geisler \& Sarajedini (1999) superimposed. Note that an age-dependent correction to the indicated metallicities, as derived in the text, was applied for Tombaugh 1.

Geisler \& Sarajedini (1999) traced standard giant branches (SGBs) in the $M_{T_{1}}$ vs. $\left(C-T_{1}\right)_{0}$ plane and demonstrated that the metallicity sensitivity of the SGBs (each giant branch corresponds to an iso-abundance curve) is three times higher than that of the $V, I$ technique (Da Costa \& Armandroff 1990). Consequently, it is possible to determine metallicities for a given photometric error, three times more precisely. Thus, we found in the SGBs a tool to roughly estimate the metallicity of Tombaugh 1 . We used the $E\left(C-T_{1}\right)$ and $T_{1}-M_{T_{1}}$ values derived from the fit of the ZAMS at $Z=0.008$ and entered the intrinsic colours and absolute magnitudes in the $M_{T_{1}}$ vs. $\left(C-T_{1}\right)_{0}$ CMD as is shown in Fig. 7. We thus derived an observed $[\mathrm{Fe} / \mathrm{H}]$ value of $-1.00 \pm 0.25 \mathrm{dex}$ for Tombaugh 1 , the relatively large uncertainty being mainly due to the large scatter of putative evolved stars in the CMD. Then, we corrected the metallicity directly read from the $M_{T_{1}}$ vs. $\left(C-T_{1}\right)_{0}$ plane in view of the effect of the well known age-metallicity degeneracy, since Geisler \& Sarajedini employed globular clusters older than $10 \mathrm{Gyr}$ for $[\mathrm{Fe} / \mathrm{H}]<-0.5 \mathrm{dex}$. We added $+0.70 \mathrm{dex}$ to our measured metallicity, which was deduced from the procedure described by Geisler et al. (2003) to correct the iron-tohydrogen ratios obtained from the SGBs. This leads to a final cluster metallicity of $[\mathrm{Fe} / \mathrm{H}]=-0.30 \pm 0.25 \mathrm{dex}$, which lends support to the metallicity estimated directly from the isochrone fitting $(Z=0.008,[\mathrm{Fe} / \mathrm{H}]=-0.40)$.

\subsection{Comparison with previous results}

As far as we know, the most extensive study on Tombaugh 1 was carried out by CP95 from CCD VI photometry of nearly 


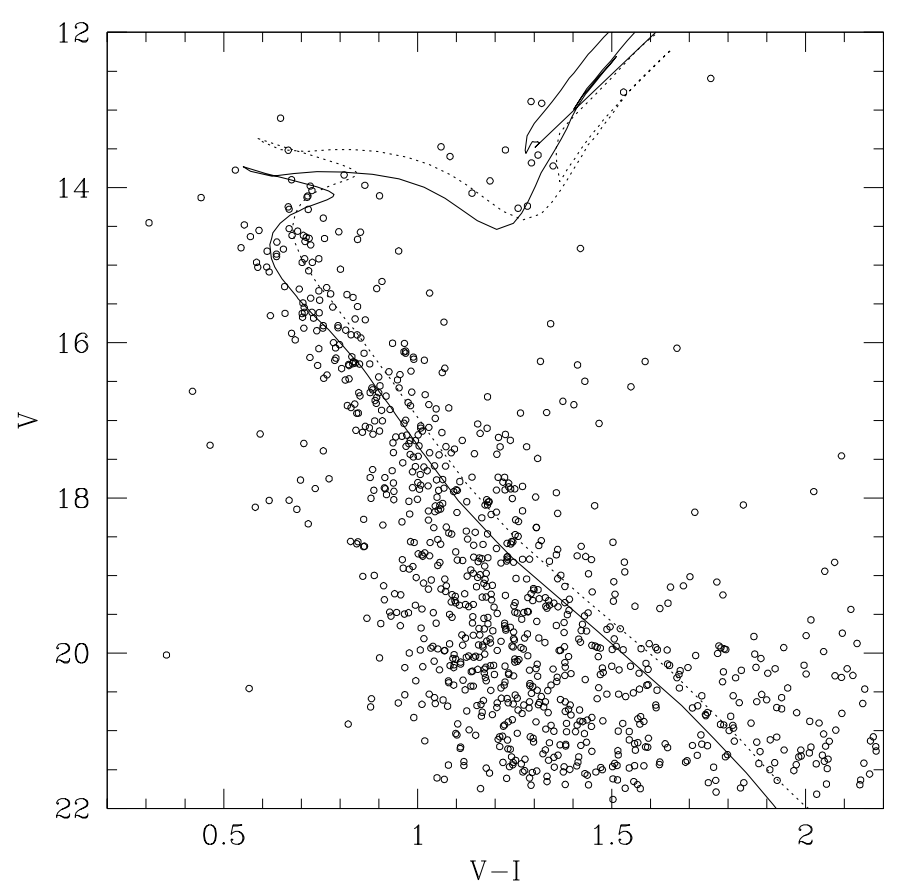

Fig. 8. Figure 7 of Carraro \& Patat (1995) with isochrones of Bertelli et al. (1994) superimposed. Solid and dashed lines correspond to isochrones of $(Z, \log t)=(0.008,9.10)$ and $(0.02,9.00)$, respectively. The isochrones were shifted by +12.70 and +0.37 in $V$ and $V-I$, which correspond to the cluster apparent distance modulus and reddening determined in Sect. 5.

a thousand of stars in the cluster field. From the fit of theoretical isochrones calculated by Bertelli et al. (1994) onto the $V$ vs. $V-I C M D$, they derived $E(B-V)=0.40 \pm 0.05$, $V-M_{V}=13.60 \pm 0.20(d=3.0 \mathrm{kpc})$ and an age of $1.0 \mathrm{Gyr}$, assuming the cluster to have solar metal content $(Z=0.020)$. Using the errors they quote for the reddening and the distance modulus, we calculated an uncertainty for their distance of $0.5 \mathrm{kpc}$. Thus, within errors range, our cluster distance and age agree with those determined by CP95. However, reddening estimates and apparent distance moduli differ significantly from one another.

With the aim of looking into the source of such difference, we re-built the $V$ vs. $V-I$ CMD of CP95 (their Fig. 7) and overplotted the isochrone of $Z=0.02$ and $\log t=9.00$ of Bertelli et al. (1994) shifted according to the here determined reddening and distance modulus. The solid line of Fig. 8 shows that our couple of values $\left(E(V-I), V-M_{V}\right)$ matches the cluster MS, from the TO region down to the faintest magnitudes, considerably better than the dashed line traced with the CP95's cluster parameters. A quick comparison of Figs. 5 and 8 allows one to conclude that the contamination of field stars below $V \approx 18$, could have misled the route of the MS traced by CP95 in the cluster CMD. Moreover, a better fit of the cluster features is obtained when the isochrone for $\log t=9.10$ and $Z=0.008$ is used, as shown in Fig. 8 (solid line).

A previous study of Tombaugh 1 was carried out by Turner (1982), who observed photoelectrically in the $U B V$ system only 26 stars in the cluster field. With the help of the colourcolour diagram, he derived a cluster reddening of $0.30 \mathrm{mag}$, which is in excellent agreement with our present estimate. However, the age derived by Turner is similar to the Hyades's age ( $\sim 600 \mathrm{Myr})$, thus resulting in nearly half the age estimated in Carraro \& Patat (1995) and in this study. We think that such difference, as well as the difference in the cluster distance Turner obtained a distance $\sim 1 / 2$ of our value - can arise from the fact that Turner used a CMD as deep as $V=15 \mathrm{mag}$, and only a crude fit of the ZAMS and of theoretical isochrones can be performed (see Turner's Fig. 5).

According to the present cluster distance, Tombaugh 1 turns out to be a relatively metal-poor intermediate-age open cluster located at $10 \mathrm{kpc}$ from the Galactic centre and at a height below the Galactic plane of $0.28 \mathrm{kpc}$. The age, the metallicity and the position in the Galaxy derived for the cluster are in complete agreement with the overall properties of the Galactic disc, i.e., the existence of a radial abundance gradient, of a predominant dispersion in the age-metallicity relation, and of no clear evidence of a gradient perpendicular to the Galactic plane (see, e.g., Piatti et al. 2003b, and references therein).

\section{Conclusions}

In this paper we present CCD photometry in the Washington system $C$ and $T_{1}$ passbands of 1351 stars in the field of the open cluster Tombaugh 1 . We have found the following main properties for the studied cluster:

- $\quad$ (i) The cluster MS in the observed $T_{1}$ vs. $C-T_{1}$ diagram, corrected by field star contamination, reveals the existence of a couple of gaps of stars at intermediate magnitudes. The overall characteristics of the cluster $T_{1}$ vs. $C-T_{1}$ diagram are compatible with the cluster's being of intermediate age. There is no evidence of differential reddening across the cluster field, the noteworthy variations of the width of the cluster MS along the $T_{1}$ magnitude being due mainly to the presence of possible binaries as well as to evolutionary effects.

- (ii) The comparison of the cluster CMD with theoretical isochrones recently computed for the Washington system by the Geneva group suggests that the cluster has a metallicity lower than the solar value. For $Z=0.008$, equivalent to $[\mathrm{Fe} / \mathrm{H}]=-0.40$ (Bertelli et al. 1994), the best-fitting isochrones yields $E\left(C-T_{1}\right)=0.55 \pm 0.10$, equivalent to $E(B-V)=0.30 \pm 0.05, T_{1}-M_{T_{1}}=12.50 \pm 0.25$, and an age of $1.3_{-0.2}^{+0.1}$ Gyr. The latter is in good agreement with that estimated from the independent metallicitiy $\delta T_{1}$ index defined by Geisler et al. (1997). Tombaugh 1 is then located at a distance of $(2.2 \pm 0.5) \mathrm{kpc}$ from the Sun and 283 pc below the Galactic plane.

- (iii) A cluster radius of $(650 \pm 50)$ pixels, equivalent to $4 ! 3 \pm 0.3$, was estimated from star counts in boxes of 100-pixel a side distributed throughout the whole field. Therefore, the cluster linear diameter turns out to be $(2.8 \pm 0.2) \mathrm{pc}$.

(iv) A metal abundance $[\mathrm{Fe} / \mathrm{H}]=-0.30 \pm 0.25$ relative to the Sun has been roughly estimated from the standard giant branches method described by Geisler \& Sarajedini (1999), in close agreement with the value derived from the 
isochrone fitting. Both the position of Tombaugh 1 in the Galaxy as well as its metal content are compatible with the existence of a radial abundance gradient in the Galactic disc. Spectroscopic analysis of the red cluster giants could greatly improve further studies of this object.

Acknowledgements. We are indebted to the CTIO staff for their hospitality and support during the observing run. We also thank the referee for his/her valuable suggestions, which have allowed us to improve the manuscript. This work was partially supported by the Argentinian institutions CONICET, SECYT (Universidad Nacional de Córdoba), Agencia Córdoba Ciencia and Agencia Nacional de Promoción Científica y Tecnológica (ANPCyT). This work is based on observations made at Cerro Tololo Inter-American Observatory, which is operated by AURA, Inc., under cooperative agreement with the NSF. We also acknowledge the use of the WEBDA database, maintained by J.-C. Mermilliod, Geneva.

\section{References}

Ahumada, J. A., \& Lapasset, E. 1995, A\&AS, 109, 375

Bertelli, G., Bressan, A., Chiosi, C., Fagotto, F., \& Nasi, E. 1994, A\&AS, 106, 275

Bergbusch, P. A., VandenBerg, D. A., \& Infante, L. 1991, AJ, 101, 2102

Bonifazi, A., Fusi Pecci, F., Romeo, G., \& Tosi, M. 1990, MNRAS, 245,15

Burki, G. 1975, A\&A, 43, 37

Canterna, R. 1976, AJ, 81, 228

Carraro, G., \& Patat, F. 1995, MNRAS, 276, 563 (CP95)

Chen, L., Hou, J. L., \& Wang, J. J. 2003, AJ, 125, 1397

Crowe, R. A., \& Mitalas, R. 1982, A\&A, 108, 55

Da Costa, G. S., \& Armandroff, T. E. 1990, AJ, 100, 162

Dutra, C. M., \& Bica, E. 2000, A\&A, 359, 347

Friel, E. D. 1995, ARA\&A, 33, 381
Friel, E. D., Janes, K. A., Tavarez, M., et al. 2002, AJ, 124, 2693

Geisler, D. 1996, AJ, 111, 480

Geisler, D., Bica, E., Dottori, H., et al. 1997, AJ, 114, 1920

Geisler, D., Lee, M. G., \& Kim, E. 1996, AJ, 111, 1529

Geisler, D., Piatti, A. E., Bica, E., \& Clariá, J. J. 2003, MNRAS, 341, 771

Geisler, D., \& Sarajedini, A. 1999, AJ, 117, 308

Girardi, L., Bertelli, G., Bressan, A., et al. 2000, A\&A, 391, 195

Haffner, H. 1957, Z. Astrophys., 43, 89

Landolt, A. U. 1992, AJ, 104, 340

Lee, M. G. 1997, AJ, 113, 729

Lejeune, T., \& Schaerer, D. 2001, A\&A, 366, 538

Mermilliod, J.-C., \& Mayor, M. 1989, A\&A, 219, 125

Mermilliod, J.-C., \& Mayor, M. 1990, A\&A, 237, 71

Ng, Y. K., Bertelli, G., Chiosi, C., \& Bressan, A. 1996, A\&A, 310, 771

Park, H. S., \& Lee, M. G. 1999, MNRAS, 304, 883

Phelps, R. L., Janes, K. A., \& Montgomery, K. A. 1994, AJ, 107, 1079

Piatti, A. E., Clariá, J. J., \& Ahumada, A. V. 2003a, MNRAS, 340, 1249

Piatti, A. E., Clariá, J. J., \& Ahumada, A. V. 2003b, MNRAS, 346, 390

Piatti, A. E., Bica, E., Geisler, D., \& Clariá, J. J. 2003c, MNRAS, 344, 965

Piatti, A. E., Clariá, J. J., \& Ahumada, A. V. 2004a, A\&A, 418, 979

Piatti, A. E., Clariá, J. J., \& Ahumada, A. V. 2004b, MNRAS, 349, 641

Ruprecht, J. 1966, Bull. Astron. Inst. Czech., 17, 33

Strobel, A. 1991, A\&A, 247, 35

Tifft, W. G. 1959, ApJ, 129, 241

Tombaugh, C. W. 1938, PASP, 50, 171

Tombaugh, C. W. 1941, PASP, 53, 219

Trumpler, R. J. 1930, Lick Obs. Bull., 14, 154

Turner, D. G. 1982, J. R. Astron. Soc. Canada, 77, 31

Turner, D. G. 1996, ASP Conf. Ser., ed. E. F. Milone, \& J. C. Mermilliod, 90, 382 